OPEN ACCESS

Edited by:

Xiaotian Wang,

Southwest University, China

Reviewed by:

Zhifeng Liu,

Inner Mongolia University, China

Cai Cheng,

Sichuan Normal University, China

Li Li,

Chongqing University of Posts and

Telecommunications, China

*Correspondence:

Shoubing Ding

shoubingding@cqnu.edu.cn

Zhimin Wu

zmwu@cqnu.edu.cn

Specialty section:

This article was submitted to

Theoretical and Computational Chemistry

a section of the journal

Frontiers in Chemistry

Received: 13 August 2020

Accepted: 07 September 2020

Published: 08 October 2020

Citation:

Chen T, Wu N, Li Y, Cui Y, Ding S and Wu Z (2020) First-Principles Study on the Magnetoelectric and Optical

Properties of Novel Magnetic

Semiconductor Li $(\mathrm{Mg}$, Cr)P With

Decoupled Spin and Charge Doping.

Front. Chem. 8:594411.

doi: 10.3389/fchem.2020.594411

\section{First-Principles Study on the Magnetoelectric and Optical Properties of Novel Magnetic Semiconductor Li(Mg, Cr)P With Decoupled Spin and Charge Doping}

\author{
Ting Chen, Nan Wu, Yue Li, Yuting Cui, Shoubing Ding * and Zhimin Wu*
}

Chongqing Key Laboratory of Photoelectric Functional Materials, College of Physics and Electronic Engineering, Chongqing Normal University, Chongqing, China

The electronic structures, magnetic and optical properties of $\mathrm{Li}_{1 \pm y}\left(\mathrm{Mg}_{1-x} \mathrm{Cr}_{x}\right) \mathrm{P}(x, y=$ $0.125)$ are calculated by using the first principles method based on density functional theory. We find that the incorporation of Cr causes the strong hybridization between Li-2s, $\mathrm{P}-2 \mathrm{p}$, and Cr-3d orbitals, resulting in a spin-polarized impurity band and forming stronger Cr-P polar covalent bonds. Li(Mgo.875 $\left.\mathrm{Cr}_{0.125}\right) \mathrm{P}$ becomes half-metallic ferromagnetism. The properties of the doped systems can be regulated by Li off-stoichiometry. When Li is deficient, the narrower impurity band and stronger $p$ - $d$ orbital hybridization enhance the half-metallicity. While the half-metallicity disappears, the band gap becomes wider, and the conductivity decreases for Li excess system, but its magnetic moments increase. Comparing optical properties show that the imaginary part of dielectric and complex refractive index function and optical absorption spectrum all have a new peak in the low energy region after $\mathrm{Cr}$ doping, and the new peaks are significantly enhanced when $\mathrm{Li}$ is deficient. The absorption range of low frequency electromagnetic wave is enlarged, and the energy loss functions show obvious red-shift effect for the doped systems. The results indicate that the properties of $\mathrm{Li}(\mathrm{Mg}, \mathrm{Cr}) \mathrm{P}$ can be controlled by $\mathrm{Cr}$ doping and $\mathrm{Li}$ off-stoichiometry independently, which will benefit potential spintronics applications.

Keywords: Cr doped LiMgP, electronic structure, ferromagnetism, optical properties, first-principles calculations

PACS: 31.15.A-, 71.15.Nc, 71.20.-b, 85.75.-d

\section{INTRODUCTION}

Diluted magnetic semiconductors (DMS) combine the charge freedom and spin freedom of the electrons in the same matrix and have both advantages of semiconductor and magnetic materials. Although a lot of studies have been carried out for the traditional DMS (Wolf et al., 2001; Zutic et al., 2004; Jungwirth et al., 2006; Dietl, 2010), there are still some obstacles that need to be solved. Firstly, for II-V-based diluted magnetic semiconductors, only local magnetic moments are introduced by replacing equivalent metal ions with $\mathrm{Mn}$. The antiferromagnetic super-exchange interaction between local magnetic moments makes them have different magnetic behaviors at different magnetic ion concentration and temperature. Secondly, for III-V-based diluted magnetic semiconductors, due to the non-equivalent substitution of doping, the solubility of the magnetic 
ions is limited and only metastable film materials can be formed (Ohno, 1998), which results in the specimens only available as thin films and sensitive to preparation methods and annealing treatments. The coupled spin and charge is an obstacle not only for fundamental understanding of ferromagnetic mechanism but also for effective improvement of controllable Curie temperature (Potashnik et al., 2001; Han et al., 2019).

These challenges have attracted great attention for finding a series of new generation DMS materials. Maŝek et al. (2007) and Deng et al. (2011) firstly through theory and experiment, respectively, discovered a kind of new diluted magnetic semiconductor $\mathrm{Li}(\mathrm{Zn}, \mathrm{Mn})$ As based on I-II-V groups. In the system, the spin is introduced by injecting $\mathrm{Mn}^{2+}$ in the site of $\mathrm{Zn}^{2+}$, and the equivalent doping makes the system has higher $\mathrm{Mn}$ solubility. The carrier concentration can be controlled by changing the content of $\mathrm{Li}$, making $\mathrm{Li}(\mathrm{Zn}, \mathrm{Mn}) \mathrm{As}$ has higher Curie temperature $\left(T_{\mathrm{c}}\right)$ than $(\mathrm{Ga}, \mathrm{Mn})$ As. Following this, Wang et al. (2014) synthesized $\mathrm{Li}_{1.1}\left(\mathrm{Zn}_{1-x} \mathrm{Cr}_{x}\right)$ As, resulting in a ferromagnetic ordering below $T_{C} \sim 218 \mathrm{~K}$, nearer to the room temperature. However, one shortcoming of $\mathrm{Mn}$-doped and $\mathrm{Cr}$ doped LiZnAs is the using of the toxic element Arsenic. The transition metal doped LiZnP (Tao et al., 2017) and LiZnN (Cui et al., 2019) show that the magnetic moments come mainly from the TM-3d orbitals. Kacimi et al. (2014) calculated the structurel, electronic and optical properties of 96 I-II-V and I-III-IV compound semiconductors by using first-principles theory and found that $\mathrm{LiMgP}$ is a direct gap semiconductor with wider band gap, which is conducive to obtaining the new DMS materials with better properties through $\mathrm{Cr}$ doping.
In this work, the electronic structures, magnetic and optical properties of $\mathrm{Li}_{1 \pm y}\left(\mathrm{Mg}_{1-x} \mathrm{Cr}_{x}\right) \mathrm{P}(x, y=0,0.125)$ are calculated by using the first principles method based on density functional theory. We find that the $\mathrm{Cr}$ doped systems exhibit halfmetallic ferromagnetism. The electrical, magnetic and optical properties of $\mathrm{Li}(\mathrm{Mg}, \mathrm{Cr}) \mathrm{P}$ can be controlled by $\mathrm{Cr}$ doping and Li off-stoichiometry independently, which will benefit potential spintronics applications.

\section{COMPUTATIONAL DETAILS}

$\mathrm{LiMgP}$ is an antifluorite structure (space group $F \overline{4} 3 \mathrm{~m}$ ) with the lattice constant $\mathrm{a}=\mathrm{b}=\mathrm{c}=6.005 \AA$ (Kuriyama et al., 1998). The anion $\mathrm{P}$ is cubic close packed, the cations $\mathrm{Mg}$ and Li fill the gaps in the tetrahedral, and the coordination numbers of anions and cations are 4 and 8 , respectively. LiMgP can be made from high temperature solid reaction between $\mathrm{Li}, \mathrm{Mg}$, and P (Kuriyama et al., 1998). LiMgP tetrahedral lattice can be viewed as a zinc blende $\mathrm{MgP}$ binary compound, filled with $\mathrm{Li}$ atoms at tetrahedral interstitial sites near P. LiMgP is a widegap semiconductor with a direct forbidden band gap of $2.43 \mathrm{eV}$ (Kuriyama et al., 1998). To our calculations, a $2 \times 1 \times 1(24$ atoms) supercell of $\mathrm{ZB}$-type $\mathrm{LiMgP}$ is constructed containing 8 $\mathrm{Li}, 8 \mathrm{Mg}$ and $8 \mathrm{P}$ atoms, as shown in Figure 1A. For the doping system, the model is constructed by replacing one $\mathrm{Mg}$ atom in the supercell with one $\mathrm{Cr}$ atom, and the doping concentration is $12.5 \%$ (Figure 1B). Besides, to study the effect of $\mathrm{Li}$ offstoichiometry on the properties of $\mathrm{Li}(\mathrm{Mg}, \mathrm{Cr}) \mathrm{P}$, the models of $\mathrm{Li}$ vacancy and excess systems also are constructed by removing or
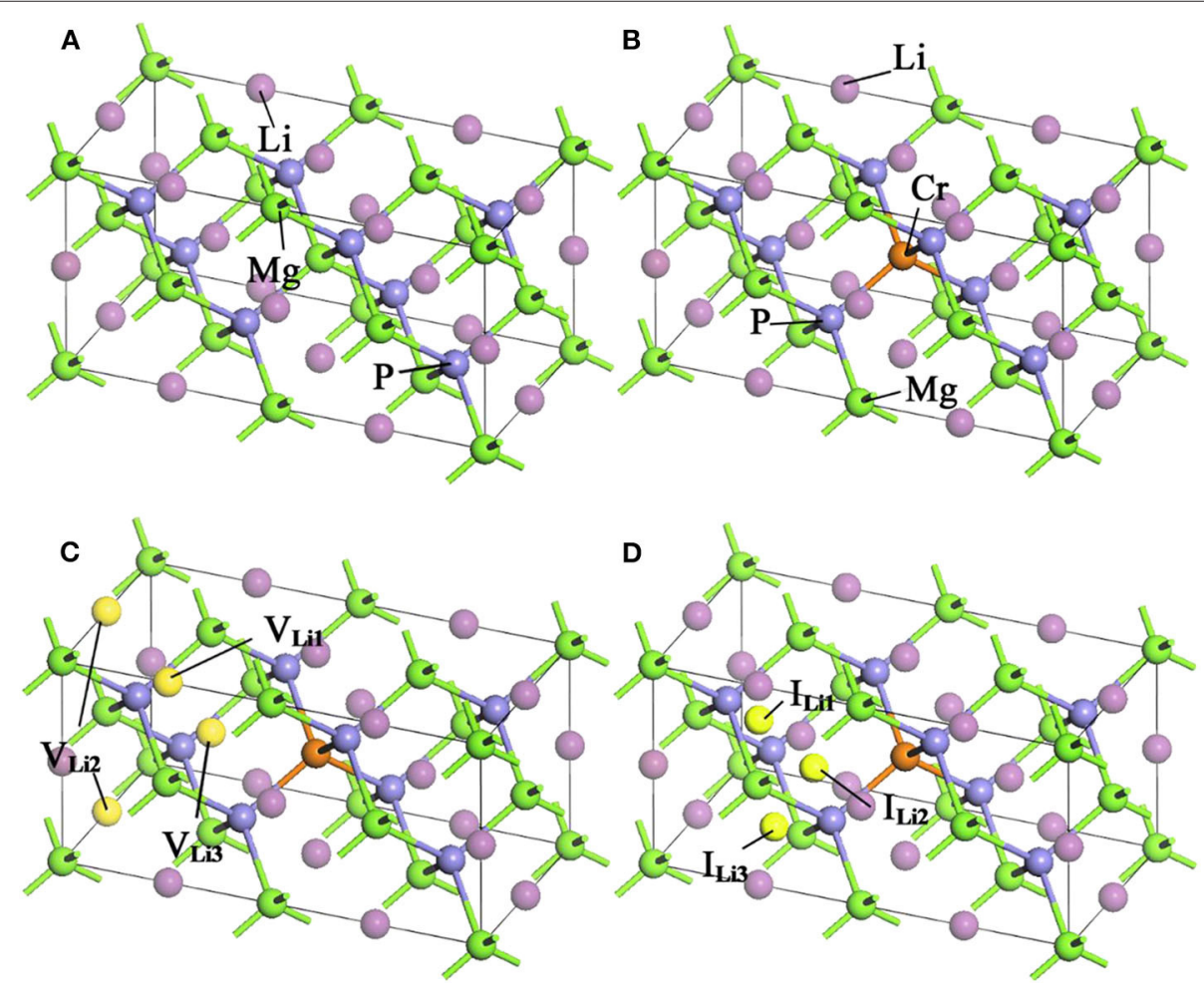

FIGURE 1 | Supercell structures of $\mathrm{Li}_{1 \pm y}\left(\mathrm{Mg}_{1-x} \mathrm{Cr}_{x}\right) \mathrm{P}$ : (A) LiMgP, (B) Li(Mgo.875 $\left.\mathrm{Cr}_{0.125}\right) \mathrm{P}$, (C) $\mathrm{Li}_{0.875}\left(\mathrm{Mg}_{0.875} \mathrm{Cr}_{0.125}\right) \mathrm{P}$, and (D) $\mathrm{Li}_{1.125}\left(\mathrm{Mg}_{0.875} \mathrm{Cr}_{0.125}\right) \mathrm{P}$. 
adding a Li atom. The different symmetry vacancy and interstitial sites $\left(\mathrm{V}_{\mathrm{Li} 1}, \mathrm{~V}_{\mathrm{Li} 2}, \mathrm{~V}_{\mathrm{Li} 3}\right.$, and $\left.\mathrm{I}_{\mathrm{Li} 1}, \mathrm{I}_{\mathrm{Li} 2}, \mathrm{I}_{\mathrm{Li} 3}\right)$ have been designated in Figures 1C,D, respectively.

The first-principles calculations are carried out with the Cambridge Serial Total Energy Package (CASTEP) code based on the density functional theory (DFT) method (Payne et al., 1992; Segall et al., 2002). The periodic boundary condition is applied in all calculations, and the generalized gradient approximation (GGA) in Perdew Burke Ernzerhof (PBE) (Perdew et al., 1996) is performed to deal with the electronic exchange-correlation potential energy. In order to reduce the number of the plane wave basis vector groups, the plane-wave ultrasoft pseudo potential (USPP) method (Vanderbilt, 1990) is implemented to describe the interaction between ionic core and valence electrons. The valence electronic configurations for $\mathrm{Li}, \mathrm{Mg}, \mathrm{P}$, and $\mathrm{Cr}$ are $\mathrm{Li}: 2 \mathrm{~s}^{1}$, Mg: $2 \mathrm{p}^{6} 3 \mathrm{~s}^{2}, \mathrm{P}: 3 \mathrm{~s}^{2} 3 \mathrm{p}^{3}$, and Cr: $3 \mathrm{~d}^{5} 4 \mathrm{~s}^{1}$, respectively. Single-particle Kohn-sham wave functions are expanded using the plane-wave with a cut-off energy of $400 \mathrm{eV}$. Sampling of the irreducible edge of the Brillouin zone is performed using the regular MonkhorstPack grid (Monkhorst and Pack, 1976) with a k-point mesh of 5 $\times 5 \times 5$. The self-consistent convergence accuracy is set at $2.0 \times$ $10^{-6} \mathrm{eV} /$ atom.

\section{RESULTS AND DISCUSSION}

\section{Structure Optimization of $\mathrm{Li}_{1 \pm y}\left(\mathrm{Mg}_{1-x} \mathrm{Cr}_{\mathrm{x}}\right) \mathrm{P}$}

To study the magnetoelectric and optical properties of novel magnetic semiconductor $\mathrm{Li}(\mathrm{Mg}, \mathrm{Cr}) \mathrm{P}$, the geometric structure of the original LiMgP supercell is firstly optimized by local Density Approximation (LDA) and General Gradient Approximation (GGA), respectively. The obtained lattice constant, band gaps and total energies are listed in Table 1, and compared with experimental data (Kuriyama et al., 1998). It can be found that the results obtained using the GGA reach a better agreement with the experimental data than those calculated using the LDA. As a consequence, it is more suitable to adopt GGA for the following calculations of $\mathrm{Li}(\mathrm{Mg}, \mathrm{Cr}) \mathrm{P}$. Besides, the geometric structures of the doping systems are also optimized with different symmetry vacancy and interstitial sites. The corresponding lattice constants, total energies and formation energies are shown in Table 2. We can find that the formation energies of $\mathrm{V}_{\mathrm{Li} 1}$ and $\mathrm{I}_{\mathrm{Li} 1}$ sites are the smallest, indicating that the doping systems of $\mathrm{V}_{\mathrm{Li} 1}$ and $\mathrm{I}_{\mathrm{Li1}}$ sites are the most stable structures in the corresponding $\mathrm{Li}$ off-stoichiometry system, respectively.

TABLE 1 | The calculated lattice constants, band gaps and total energies of LiMgP with LDA and GGA.

\begin{tabular}{lccccc}
\hline LiMgP & $\boldsymbol{a ( \AA )}$ & $\boldsymbol{b}(\AA)$ & $\boldsymbol{c}(\mathbf{A})$ & $\begin{array}{c}\text { Band } \\
\mathbf{g a p}\left(\boldsymbol{E}_{\mathbf{g}}\right) / \mathbf{e V}\end{array}$ & $\begin{array}{c}\text { Total } \\
\text { energy/eV }\end{array}$ \\
\hline $\begin{array}{l}\text { Experiment value } \\
\text { (Kuriyama et al., 1998) }\end{array}$ & 6.005 & 6.005 & 12.010 & 2.43 & - \\
LDA & 5.904 & 5.904 & 11.811 & 1.365 & $-10,754.55$ \\
GGA & 6.030 & 6.030 & 12.061 & 1.533 & $-10,766.57$
\end{tabular}

\section{Electronic Structure of $\mathrm{Li}_{1 \pm y}\left(\mathrm{Mg}_{1-x} \mathrm{Cr}_{\mathrm{x}}\right) \mathrm{P}$}

The spin polarized band structures of $\mathrm{Li}_{1 \pm y}\left(\mathrm{Mg}_{1-x} \mathrm{Cr}_{x}\right) \mathrm{P}$ are shown in Figure 2. The insets are enlarged views of the vicinity of the Fermi energy level. We can find in Figures 2A,B that both the valence band maxima and the conduction band minima are at the high symmetry $\Gamma$ point of Brillouin-zone, indicating that LiMgP is a direct gap semiconductor. The band structures of majorityspin and minority-spin are symmetrical completely, implying that the system has no net magnetic moments. The calculated band gap for LiMgP is $1.533 \mathrm{eV}$ (shown in Table 3), which is smaller than the experimental result of $2.43 \mathrm{eV}$ (Kuriyama et al., 1998). This is not surprising as the underestimation of the band gap is due to the generic nature of the density functional theory (Perdew and Levy, 1983; Godby et al., 1988). Nevertheless, this has no effect on the investigation of the electronics structure and relevant properties for Cr doped LiMgP systems (Shang et al., 2004).

As shown in Figures 2C,D, when $\mathrm{Cr}$ doped, the conduction band minima occur at the $\Gamma$ point, while the valence band maxima are located around the $\mathrm{Z}$ point, in the Brillouin Zone. This result indicates that the material transforms into an indirect semiconductor. The band gap of $\mathrm{Li}\left(\mathrm{Mg}_{0.875} \mathrm{Cr}_{0.125}\right) \mathrm{P}$ is $2.544 \mathrm{eV}$ (Table 3), which obviously increases compared with that of LiMgP. This is because ten new spin impurity levels are emerged in band gap after doping Cr. Among them, each of the majority-spin and minority-spin bands has five impurity levels. The majority-spin impurity bands slightly cross the Femi level, demonstrating that the majority-spin bands exhibit metallic properties. While the minority-spin bands reveal still semi-conductive natures, making $\mathrm{Li}\left(\mathrm{Mg}_{0.875} \mathrm{Cr}_{0.125}\right) \mathrm{P}$ system become a half-metallic material with $100 \%$ spin-polarized ratio of conduction electron. The spin-flip band gap is $0.767 \mathrm{eV}$, which are much larger than many other 3d-transition-metal-element-based materials (Guo et al., 2016). Usually, the larger spin-flip band gap, the more robust half-metallic behavior to lattice deformation and temperature (Guo et al., 2016). So the Cr doped LiMgP compounds reported in this work can be regarded as the good candidates for spintronics devices due to their large spin-flip gaps and robust half-metallicity.

$\mathrm{Li}_{0.875}\left(\mathrm{Mg}_{0.875} \mathrm{Cr}_{0.125}\right) \mathrm{P}$ is also an indirect band gap semiconductor with the band gap of $2.531 \mathrm{eV}$ (as show in

TABLE 2 | The lattice constants, total energies and formation energies of $\mathrm{Li}_{1 \pm y}\left(\mathrm{Mg}_{1-x} \mathrm{Cr}_{x}\right) \mathrm{P}$.

\begin{tabular}{lcccccc}
\hline Structure cell & \multicolumn{3}{c}{ lattice constants (Å) } & $\begin{array}{c}\text { Total } \\
\text { energy(eV) }\end{array}$ & $\begin{array}{c}\text { Formation } \\
\text { energy(eV) }\end{array}$ \\
\cline { 2 - 5 } & $\boldsymbol{a}$ & $\boldsymbol{b}$ & $\boldsymbol{c}$ & & \\
\hline $\mathrm{Li}\left(\mathrm{Mg}_{0.875} \mathrm{Cr}_{0.125}\right) \mathrm{P}$ & 6.005 & 6.005 & 12.010 & $-10,445.25$ & -6.569 \\
$\mathrm{Li}_{0.875}\left(\mathrm{Mg}_{0.875} \mathrm{Cr}_{0.125}\right) \mathrm{P}-1$ & 5.999 & 5.999 & 11.885 & $-12,067.47$ & -3.513 \\
$\mathrm{Li}_{0.875}\left(\mathrm{Mg}_{0.875} \mathrm{Cr}_{0.125}\right) \mathrm{P}-2$ & 5.990 & 6.005 & 11.912 & $-12,067.18$ & -3.221 \\
$\mathrm{Li}_{0.875}\left(\mathrm{Mg}_{0.875} \mathrm{Cr}_{0.125}\right) \mathrm{P}-3$ & 5.986 & 5.986 & 11.919 & $-12,067.19$ & -3.234 \\
$\mathrm{Li}_{1.125}\left(\mathrm{Mg}_{0.875} \mathrm{Cr}_{0.125}\right) \mathrm{P}-1$ & 6.038 & 6.0378 & 12.025 & $-12,448.96$ & -8.911 \\
$\mathrm{Li}_{1.125}\left(\mathrm{Mg}_{0.875} \mathrm{Cr}_{0.125}\right) \mathrm{P}-2$ & 6.035 & 6.043 & 12.039 & $-12,448.71$ & -8.659 \\
$\mathrm{Li}_{1.125}\left(\mathrm{Mg}_{0.875} \mathrm{Cr}_{0.125}\right) \mathrm{P}-3$ & 6.036 & 6.042 & 12.039 & $-12,448.71$ & -8.659
\end{tabular}



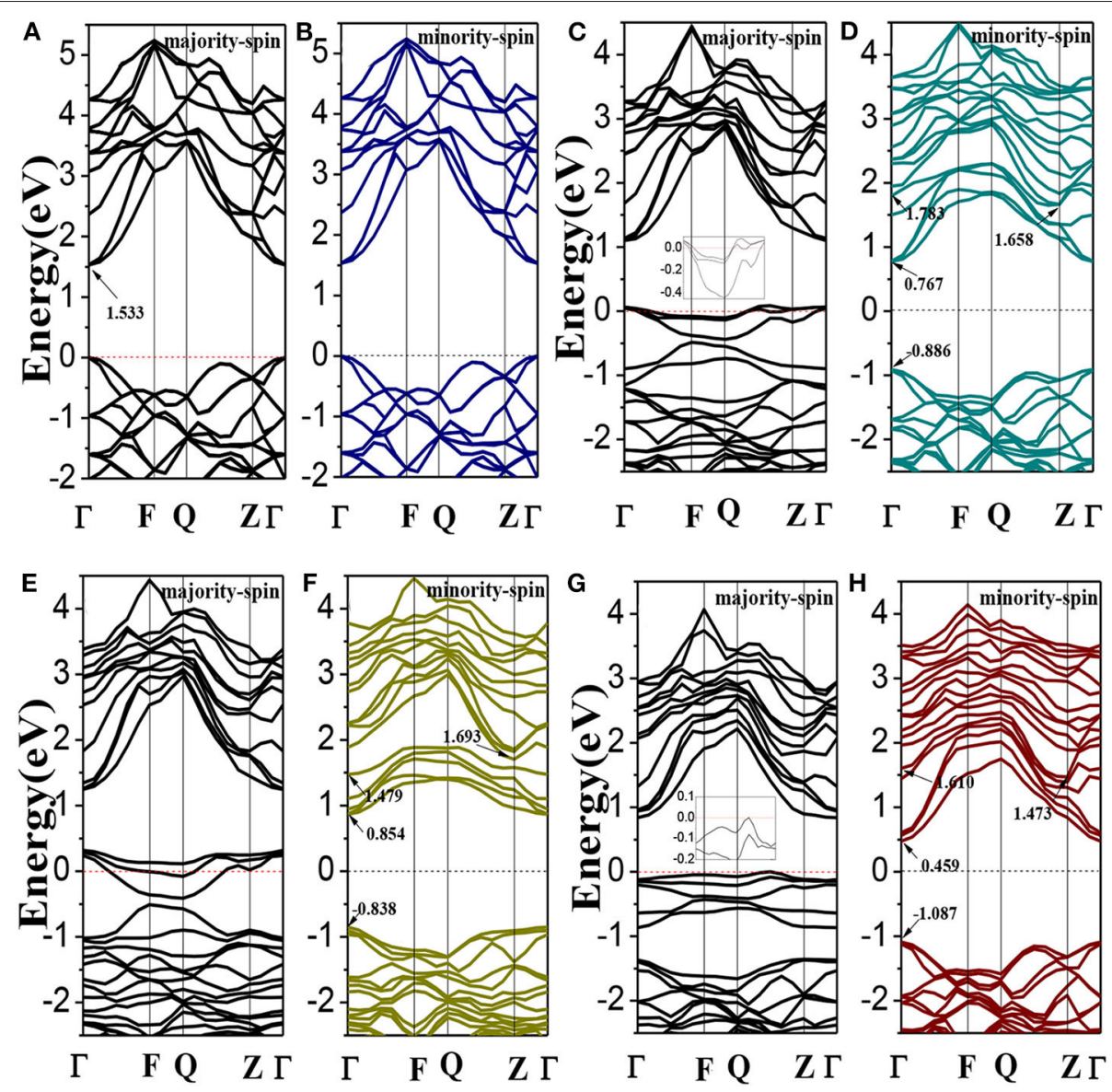

FIGURE 2 | The band structures of $\mathrm{Li}_{1 \pm y}\left(\mathrm{Mg}_{1-x} \mathrm{Cr}_{x}\right) \mathrm{P}$ : (A,B) LiMgP, (C,D) Li(Mgo.875 $\left.\mathrm{Cr}_{0.125}\right) \mathrm{P}, \mathbf{( E , F ) ~ L i ~} i_{0.875}\left(\mathrm{Mg}_{0.875} \mathrm{Cr}_{0.125}\right) \mathrm{P}$, and $(\mathbf{G}, \mathrm{H}) \mathrm{Li}_{1.125}\left(\mathrm{Mg}_{0.875} \mathrm{Cr}_{0.125}\right) \mathrm{P}$. Inset: enlarged views of the vicinity of the Fermi energy level.

TABLE 3 | The band gaps, impurity band widths, half-metallic gap and magnetic moments of $\mathrm{Li}_{1 \pm y}\left(\mathrm{Mg}_{1-x} \mathrm{Cr}_{x}\right) \mathrm{P}$.

\begin{tabular}{lcccc}
\hline $\mathrm{Li}_{1 \pm y}\left(\mathbf{M g}_{1-x} \mathrm{Cr}_{\mathbf{x}}\right) \mathbf{P}$ & $\begin{array}{c}\text { Band } \\
\text { gap }\left(\mathrm{E}_{\mathbf{g}}\right) / \mathrm{eV}\end{array}$ & $\begin{array}{c}\text { Impurity } \\
\text { band } \\
\text { width/eV }\end{array}$ & $\begin{array}{c}\text { half-metallic } \\
\text { gap/eV }\end{array}$ & $\begin{array}{c}\text { Magnetic } \\
\text { moment } / \mu_{\mathbf{B}}\end{array}$ \\
\hline $\mathrm{LiMgP}$ & 1.533 & - & - & 0 \\
$\mathrm{Li}\left(\mathrm{Mg}_{0.875} \mathrm{Cr}_{0.125}\right) \mathrm{P}$ & 2.544 & 1.016 & 0.767 & 4.08 \\
$\mathrm{Li}_{0.875}\left(\mathrm{Mg}_{0.875} \mathrm{Cr}_{0.125}\right) \mathrm{P}$ & 2.531 & 0.625 & 0.854 & 2.98 \\
$\mathrm{Li}_{1.125}\left(\mathrm{Mg}_{0.875} \mathrm{Cr}_{0.125}\right) \mathrm{P}$ & 2.560 & 1.151 & - & 4.90
\end{tabular}

Figures 2E,F). Three majority-spin impurity bands cross the Fermi level exhibiting a metallic nature, while the minority-spin impurity bands are above the Fermi level, resulting in that the Li vacancy system also exhibits a half-metallic nature. The spin-flip band gap of $\mathrm{Li}_{0.875}\left(\mathrm{Mg}_{0.875} \mathrm{Cr}_{0.125}\right) \mathrm{P}$ is $0.854 \mathrm{eV}$, and the half-metallicity is enhanced obviously when Li is deficient. Li excess system is also an indirect band gap semiconductor with the band gap of $2.560 \mathrm{eV}$. However, neither of the majority-spin and minority-spin impurity bands crosses the
Fermi level. Therefore, $\mathrm{Li}_{1.125}\left(\mathrm{Mg}_{0.875} \mathrm{Cr}_{0.125}\right) \mathrm{P}$ change back to semiconducting nature again.

The calculated total and partial density of states (DOS) for $\mathrm{Li}_{1 \pm y}\left(\mathrm{Mg}_{1-x} \mathrm{Cr}_{x}\right) \mathrm{P}$ are shown in Figure 3. For pure LiMgP, we can find that there are two peaks in the valence band of TDOS ( 1 and 2 in Figure 3A). Peak 1 is mainly contributed by the electrons of $\mathrm{Mg}-3 \mathrm{~s}$ and $\mathrm{P}-3 \mathrm{p}$ states and peak 2 mainly contains the electrons of Li-2s, Mg-2p, and P-3p states. The conduction band is mainly composed of the electrons of Li-2s, Mg-2p, and $\mathrm{Mg}-3 \mathrm{~s}$, and the Mg-2p states. The states of spin-up and spindown are well-symmetry, revealing that pure LiMgP has no net magnetic moment. For Cr doped system, Li-2s, P-3p, and Cr-3d orbitals hybridize near the Fermi energy (Figure 3B), resulting in that the $t_{2 \mathrm{~g}}$ and $e_{\mathrm{g}}$ energy levels of $\mathrm{Cr}-3 d$ state are separated from each other, and the $t_{2 \mathrm{~g}}$ energy level is pushed toward the Fermi level, induce $\mathrm{Li}\left(\mathrm{Mg}_{0.875} \mathrm{Cr}_{0.125}\right) \mathrm{P}$ to become half-metallic ferromagnetism. When $\mathrm{Li}$ is deficient, the electrons of $\mathrm{P}-3 \mathrm{p}$ and $\mathrm{Cr}-3 \mathrm{~d}$ states have the stronger $\mathrm{p}-\mathrm{d}$ orbitals hybridization (Figure 3C). The $t_{2 \mathrm{~g}}$ levels are pushed up even further, making its occupation states reduce from three to one, which causes that the spin-flip band gap of $\mathrm{Li}_{0.875}\left(\mathrm{Mg}_{0.875} \mathrm{Cr}_{0.125}\right) \mathrm{P}$ increases. When $\mathrm{Li}$ is excess, only a weak hybridization of $\mathrm{p}$ - $\mathrm{d}$ orbitals appears near 

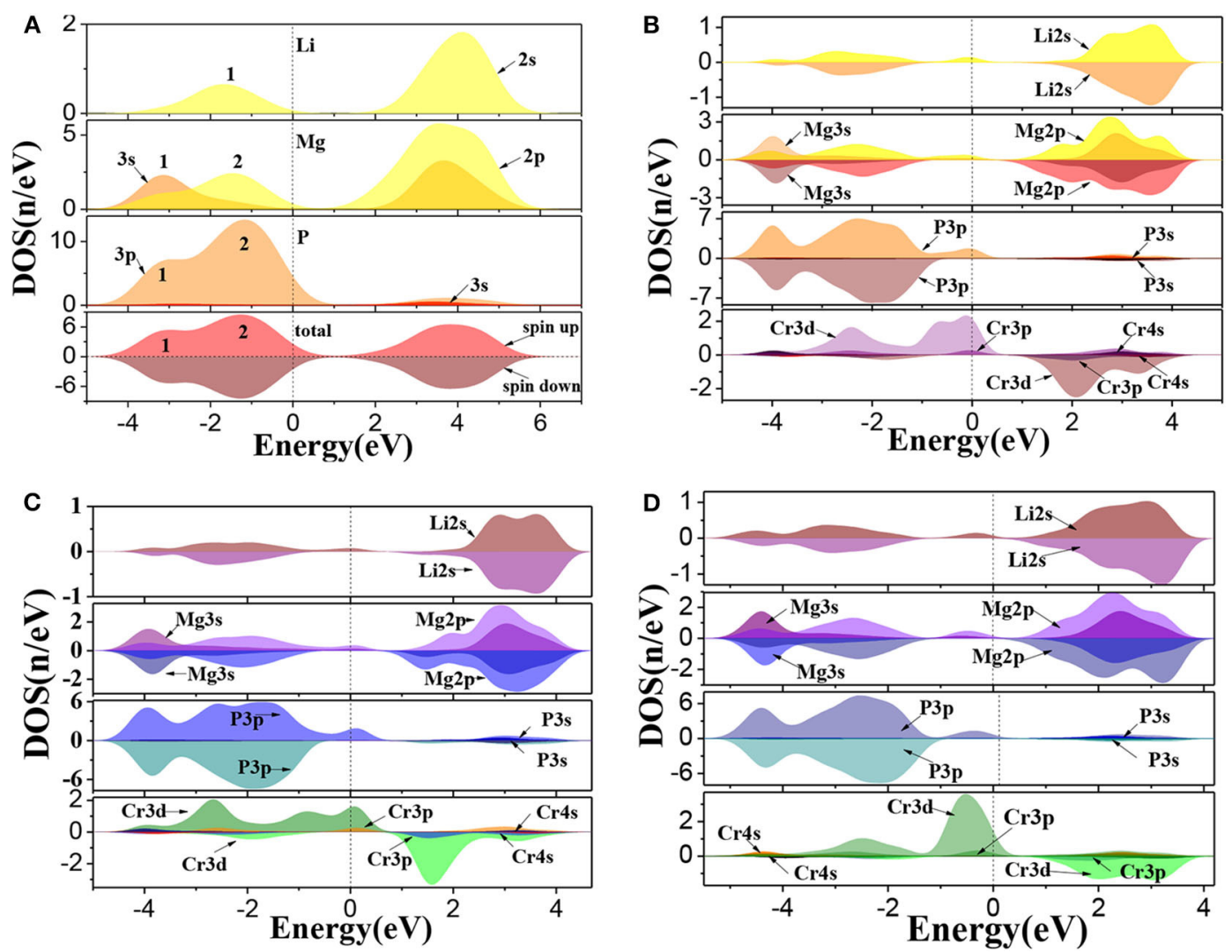

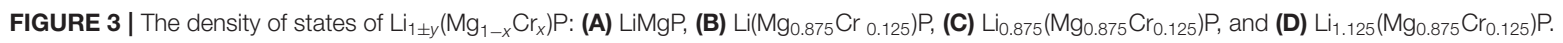

the Fermi energy, and the $t_{2 \mathrm{~g}}$ levels is completely occupied by electrons. The half-metallicity disappears.

Moreover, the net magnetic moment is also calculated by integrating the occupied states below the Fermi energy and shown in Table 3. The obtained net magnetic moments are 4.08 $\mu_{\mathrm{B}}, 2.98 \mu_{\mathrm{B}}$, and $4.90 \mu_{\mathrm{B}}$ for the Cr doped, Li vacancy, and $1 \mathrm{Li}$ excess system, respectively. The result indicates that the impurity band width and the net magnetic moments increase with the increasing of Li concentration.

Figure 4 shows the plots of charge density difference for $\mathrm{Li}_{1 \pm y}\left(\mathrm{Mg}_{1-x} \mathrm{Cr}_{x}\right) \mathrm{P}$. For pure LiMgP (Figure 4a), the electron cloud of the $\mathrm{P}$ atom close to the $\mathrm{Mg}$ atom is denser. The second orbital of the $\mathrm{P}$ atom is polarized, and the electrons move inward. The $\mathrm{Mg}$ and $\mathrm{P}$ atoms form polarized covalent bonds. When $\mathrm{Cr}$ doped (Figure $4 \mathrm{~b}$ ), the $\mathrm{P}$ atoms gain more electrons from $\mathrm{Cr}$ atoms than the $\mathrm{Mg}$ atoms, indicating that the $\mathrm{Cr}-\mathrm{P}$ polarized covalent bonds are stronger than that those of the Mg-P, which can be attributed to the strong hybridization between Li-2s, P$2 \mathrm{p}$, and $\mathrm{Cr}-3 \mathrm{~d}$ orbitals. When $\mathrm{Li}$ is deficient, the charge density between $\mathrm{Li}$ and $\mathrm{P}$ atoms becomes weaker, meanwhile, the charge density between $\mathrm{Cr}$ and $\mathrm{P}$ atoms turn stronger, indicating that $\mathrm{P}$ atoms cannot gain electrons from $\mathrm{Li}$ atoms, so the more charges need to be gained from $\mathrm{Cr}$ atoms. It can be seen in Figure $4 \mathbf{d}$ that $\mathrm{P}$ atoms can gain more electrons from $\mathrm{Li}$ atoms, resulting in that the charge loss of $\mathrm{Cr}$ atoms becomes less and the $\mathrm{Cr}-\mathrm{P}$ polar covalent bonds become weaker for Li excess system.

\section{Optical Properties of $\mathrm{Li}_{1 \pm y}\left(\mathbf{M g}_{1-x} \mathrm{Cr}_{x}\right) \mathrm{P}$}

To further investigate the effects of $\mathrm{Cr}$ doping and $\mathrm{Li}$ offstoichiometry on properties of $\mathrm{Li}_{1 \pm y}\left(\mathrm{Mg}_{1-x} \mathrm{Cr}_{x}\right) \mathrm{P}$, the imaginary part of dielectric functions, complex refractive index functions, optical absorption spectra and energy loss spectra are also calculated and shown in Figure 5. We can find from Figure 5A that there is a dielectric peak at $E=4.27 \mathrm{eV}$, corresponding to the direct transition of $\mathrm{P}-3 \mathrm{p}$ in valence bands to $\mathrm{Li}-2 \mathrm{~s}, \mathrm{Mg}$ 3s, and $\mathrm{Mg}-2 \mathrm{p}$ states in conduction bands. It is worth noting that a new peak occurs in the low energy region and the peak at $E=4.27 \mathrm{eV}$ reduces after $\mathrm{Cr}$ doping. Moreover, we also find that the new peak is significantly enhanced when $\mathrm{Li}$ is deficient, but when Li is excess the new peak disappears, and the main peak moves slightly toward the low energy region. The refractive index for pure $\mathrm{LiMgP}$ is 2.571 (Figure 5B). In the lower energy $(E<1.82 \mathrm{eV})$ and higher energy $(E>11.54 \mathrm{eV})$ region, the imaginary part of the complex refractive index function is zero, and the real part is a constant, indicating that the absorption of $\mathrm{LiMgP}$ is limit to a certain frequency range. Similarly to the dielectric functions, there are also new peaks in the low energy region of complex refractive index functions after $\mathrm{Cr}$ doping, implying that the absorption range of low frequency electromagnetic wave is enlarged for the doped systems. It can be seen in Figure 5C that a new peak of the optical absorption spectrum also appears in the low energy region after Cr doping, the main peak of absorption spectrum moves slightly toward 


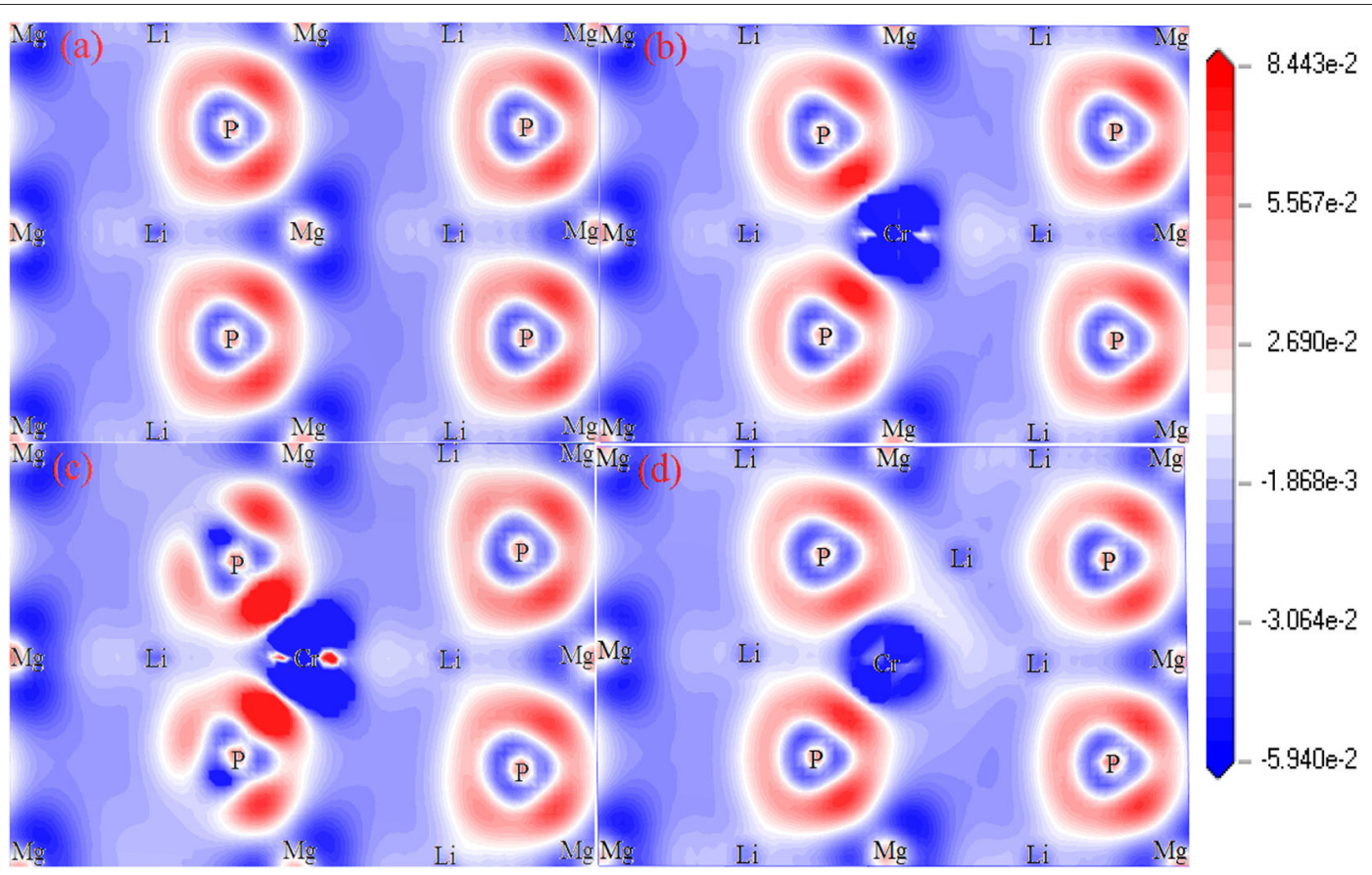

FIGURE 4 | The charge density difference of $\mathrm{Li}_{1 \pm y}\left(\mathrm{Mg}_{1-x} \mathrm{Cr}_{x}\right) \mathrm{P}$ : (a) $\mathrm{LiMgP}$, (b) $\mathrm{Li}\left(\mathrm{Mg}_{0.875} \mathrm{Cr}_{0.125}\right) \mathrm{P}$, (c) $\mathrm{Li}_{0.875}\left(\mathrm{Mg}_{0.875} \mathrm{Cr}_{0.125}\right) \mathrm{P}$, and (d) $\mathrm{Li}_{1.125}\left(\mathrm{Mg}_{0.875} \mathrm{Cr}_{0.125}\right)$.
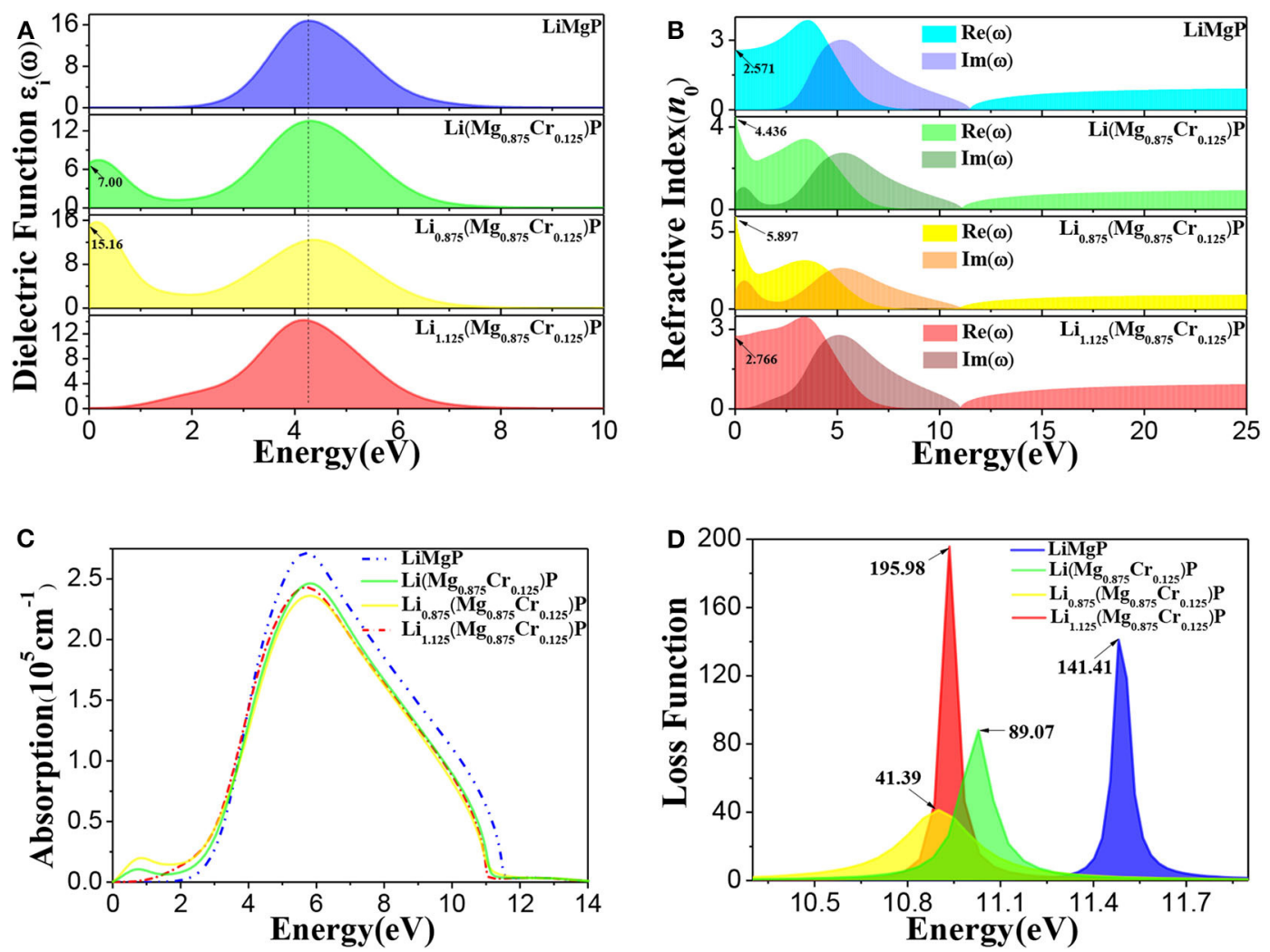

FIGURE 5 | The optical properties of $\mathrm{Li}_{1 \pm y}\left(\mathrm{Mg}_{1-x} \mathrm{Cr}\right)_{x} \mathrm{P}$ : (A) imaginary part of dielectric functions, (B) complex refractive index functions, (C) optical absorption spectra, (D) energy loss spectra. 
the low energy region, demonstrating again that the absorption of low frequency electromagnetic wave is enhanced. As shown in Figure 5D, the energy loss functions also move obviously toward the low energy region, implying the red-shift effect for the doped systems. The energy loss for $\mathrm{Li}\left(\mathrm{Mg}_{0.875} \mathrm{Cr}_{0.125}\right) \mathrm{P}$, $\mathrm{Li}_{0.875}\left(\mathrm{Mg}_{0.875} \mathrm{Cr}_{0.125}\right) \mathrm{P}$, and $\mathrm{Li}_{1.125}\left(\mathrm{Mg}_{0.875} \mathrm{Cr}_{0.125}\right) \mathrm{P}$ are about $63 \%, 29 \%$ and $138 \%$ of that for LiMgP, respectively.

\section{SUMMARY}

The electronic structures, magnetic and optical properties of $\mathrm{Li}_{1 \pm y}\left(\mathrm{Mg}_{1-x} \mathrm{Cr}_{x}\right) \mathrm{P}(x, y=0,0.125)$ are calculated by using the first principles method based on density functional theory. We find that the $\mathrm{Cr}$ doped systems exhibit the halfmetallic ferromagnetism. Due to the Cr doping, sp-d orbitals hybridization leads to spin-polarized impurity bands and form stronger Cr-P polar covalent bonds. Moreover, we also find that the properties of the doped systems can be regulated by Li offstoichiometry. The impurity band width and the net magnetic moment increase with the increasing of Li concentration, but the half-metallicity and the conductivity decrease. When $\mathrm{Li}$ is excess, the p-d orbitals hybridization obviously becomes weaker and the half-metallicity disappears. Comparing optical properties shows that the imaginary part of dielectric, the complex refractive index function and the optical absorption spectrum all have

\section{REFERENCES}

Cui, Y., Zhu, J. G., Tao, H. L., Liu, S. M., Lv, Y. Z., He, M., et al. (2019). Magnetic properties of diluted magnetic semiconductors $\mathrm{Li}(\mathrm{Zn}, \mathrm{TM}) \mathrm{N}$ with decoupled charge and spin doping (TM: V, Cr, Mn, Fe, Co and Ni). Comp. Mater. Sci. 158, 260-264. doi: 10.1016/j.commatsci.2018.11.022

Deng, Z., Jin, C. Q., Liu, Q. Q., Wang, X. C., Zhu, J. L., Feng, S. M., et al. (2011). $\mathrm{Li}(\mathrm{Zn}, \mathrm{Mn}) \mathrm{As}$ as a new generation ferromagnet based on a I-II-V semiconductor. Nat. Commun. 2:422. doi: 10.1038/ncomms 1425

Dietl, T. (2010). A ten-year perspective on dilute magnetic semiconductors and oxides. Nat. Mater. 9, 965-974. doi: 10.1038/nmat2898

Godby, R. W., Schluter, M., and Sham, L. J. (1988). Self-energy operators and exchange-correlation potentials in semiconductors. Phys. Rev. B 37, 10159-10175. doi: 10.1103/PhysRevB.37.10159

Guo, R. K., Liu, G. D., Wang, X. T., Rozale, H., Wang, L. Y., Khenata, R., et al. (2016). First-principles study on quaternary Heusler compounds $\mathrm{ZrFeVZ}$ $(\mathrm{Z}=\mathrm{Al}, \mathrm{Ga}, \mathrm{In})$ with large spin-flip gap. RSC Adv. 6, 109394-109400. doi: 10.1039/C6RA18873G

Han, W., Chen, B. J., Gu, B., Zhao, G. Q., Yu, S., Wang, X. C., et al. (2019). Li(Cd,Mn)P: a new cadmium based diluted ferromagnetic semiconductor with independent spin \& charge doping. Sci. Rep. 9:7490. doi: 10.1038/s41598-019-43754-x

Jungwirth, T., Sinova, J., Masek, J., Kucera, J., and MacDonald, A. H. (2006). Theory of ferromagnetic (III,Mn)V semiconductors. Rev. Mod. Phys. 78, 809-864. doi: 10.1103/RevModPhys.78.809

Kacimi, S., Mehnane, H., and Zaoui, A. (2014). I-II-V and I-III-IV half-Heusler compounds for optoelectronic applications: comparative ab initio study. J. Alloy. Compd. 587, 451-458. doi: 10.1016/j.jallcom.2013.10.046

Kuriyama, K., Kushida, T., and Taguchi, R. (1998). Optical band gap of the ordered filled-tetrahedral semiconductor LiMgP. Solid State Commun. 108, 429-432. doi: 10.1016/S0038-1098(98)00384-6

Maŝek, J., Kudrnovsky, J., Maca, F., Gallagher, B. L., Campion, R. P., Gregory, D. H., et al. (2007). Dilute moment n-type ferromagnetic semiconductor Li(Zn,Mn)As. Phys. Rev. Lett. 98:067202. doi: 10.1103/PhysRevLett.98.067202 a new peak in the low energy region after $\mathrm{Cr}$ doping, and the new peaks are significantly enhanced when $\mathrm{Li}$ is deficient. The absorption range of low frequency electromagnetic wave is enlarged, and the energy loss functions show obvious redshift effect for the doped systems. The results indicate that the properties of $\mathrm{Li}(\mathrm{Mg}, \mathrm{Cr}) \mathrm{P}$ can be controlled by $\mathrm{Cr}$ doping and Li off-stoichiometry independently, which will benefit potential spintronics applications.

\section{DATA AVAILABILITY STATEMENT}

The raw data supporting the conclusions of this article will be made available by the authors, without undue reservation.

\section{AUTHOR CONTRIBUTIONS}

All authors listed have made a substantial, direct and intellectual contribution to the work, and approved it for publication.

\section{FUNDING}

The work described in this paper was supported by Chongqing Natural Science Foundation of China (Grant No. cstc2019jcyjmsxmX0251).
Monkhorst, H. J., and Pack, J. D. (1976). Special points for brillouin-zone integrations. Phys. Rev. B 13, 5188-5192. doi: 10.1103/PhysRevB.13.5188

Ohno, H. (1998). Making nonmagnetic semiconductors ferromagnetic. Science 281, 951-956. doi: 10.1126/science.281.5379.951

Payne, M. C., Teter, M. P., Allan, D. C., Arias, T. A., and Joannopoulos, J. D. (1992). Iterative minimization techniques for abinitio total-energy calculations molecular dynamics and conjugate gradients. Rev. Mod. Phys. 64, 1045-1097. doi: 10.1103/RevModPhys.64.1045

Perdew, J. P., Burke, K., and Ernzerhof, M. (1996). Generalized gradient approximation made simple. Phys. Rev. Lett. 77, 3865-3868. doi: 10.1103/PhysRevLett.77.3865

Perdew, J. P., and Levy, M. (1983). Physical content of the exact KohnSham orbital energies band gaps and derivative discontinuities. Phys. Rev. Lett. 51, 1884-1887. doi: 10.1103/PhysRevLett.5 1.1884

Potashnik, S. J., Ku, K. C., Chun, S. H., Berry, J. J., Samarth, N., and Schiffer, P. (2001). Effects of annealing time on defect-controlled ferromagnetism in $\mathrm{Ga}_{1-x} \mathrm{Mn}_{x}$ As. Appl. Phys. Lett. 79, 1495-1497. doi: 10.1063/1.13 98619

Segall, M. D., Lindan, P. J. D., Probert, M. J., Pickard, C. J., Hasnip, P. J., Clark, S. J., et al. (2002). First-principles simulation: ideas, illustrations and the CASTEP code. J. Phys. Condens. Mat. 14, 2717-2744. doi: 10.1088/0953-8984/14/ $11 / 301$

Shang, G., Peacock, P. W., and Robertson, J. (2004). Stability and band offsets of nitrogenated high-dielectric-constant gate oxides. Appl. Phys. Lett. 84:106. doi: $10.1063 / 1.1638896$

Tao, H. L., Wang, M. X., Zhang, Z. H., He, M., and Song, B. (2017). Effects of transition metal $(\mathrm{TM}=\mathrm{V}, \mathrm{Cr}, \mathrm{Mn}, \mathrm{Fe}, \mathrm{Co}$, and $\mathrm{Ni}$ ) elements on magnetic mechanism of LiZnP with decoupled charge and spin doping. J. Supercond. Nov. Magn. 30, 2823-2828. doi: 10.1007/s10948-0174037-1

Vanderbilt, D. (1990). Soft self-consistent pseudopotentials in a generalized eigenvalue formalism. Phys. Rev. B 41, 7892-7895. doi: 10.1103/PhysRevB.41.7892 
Wang, Q., Man, H. Y., Ding, C., Gong, X., Guo, S. L., Jin, H. K., et al. (2014). $\mathrm{Li}_{1.1}\left(\mathrm{Zn}_{1-x} \mathrm{Cr}_{x}\right)$ As: $\mathrm{Cr}$ doped I-II-V diluted magnetic semiconductors in bulk form. J. Appl. Phys. 115:083917. doi: 10.1063/1.48 67299

Wolf, S. A., Awschalom, D. D., Buhrman, R. A., Daughton, J. M., von Molnar, S., Roukes, M. L., et al. (2001). Spintronics: A spin-based electronics vision for the future. Science 294, 1488-1495. doi: 10.1126/science.10 65389

Zutic, I., Fabian, J., and Das Sarma, S. (2004). Spintronics: fundamentals and applications. Rev. Mod. Phys. 76, 323-410. doi: 10.1103/RevModPhy s.76.323
Conflict of Interest: The authors declare that the research was conducted in the absence of any commercial or financial relationships that could be construed as a potential conflict of interest.

Copyright $\odot 2020$ Chen, Wu, Li, Cui, Ding and Wu. This is an open-access article distributed under the terms of the Creative Commons Attribution License (CC BY). The use, distribution or reproduction in other forums is permitted, provided the original author(s) and the copyright owner(s) are credited and that the original publication in this journal is cited, in accordance with accepted academic practice. No use, distribution or reproduction is permitted which does not comply with these terms. 